\title{
Study on the Photocatalytic Removal of Styrene in Water
}

\author{
C. LIU $^{1}$ \\ ${ }^{1}$ Environmental Protection and Energy Saving Center, China Waterborne Transport Research \\ Institute, Beijing 100088, China
}

*Corresponding author: liuchen@wti.ac.cn, xpshino1@126.com

Keywords: Photo-catalyst, $\mathrm{TiO}_{2}$, Styrene

\begin{abstract}
With the development of economy in China, especially energy, petrochemical industry, the amount of water transportation of chemicals is increasing day by day. At the same time, the chemical leakage probability is greatly increased, and it also affects people's daily life and health. In this study, we modify the titanium dioxide using other materials and the light utilization rate and photocatalytic activity of $\mathrm{TiO}_{2}$ is improved. We also apply it in the disposal of styrene in the river water and achieved good results. In addition, the catalytic effect of various catalysts was compared, and the effect of different conditions to the reaction is also researched.
\end{abstract}

\section{Introduction}

As the development of economy in China, especially energy, petrochemical industry, the amount of water transportation of chemicals is increasing day by day. At the same time, the chemical leakage probability is greatly increased which affects people's daily life and health. In order to control and manage all kinds of pollutants to the environment, governments and researchers have made a lot of efforts to develop new technologies.

In last century, the Japanese researcher found that the titanium dioxide could decompose water. From that time, photocatalytic materials have been attracted great interest. In order to improve the photocatalytic efficiency of photocatalyst, many scientists have carried out a lot of research and exploration. Among them, there are some common photocatalysts, such as $\mathrm{TiO}_{2}, \mathrm{CdS}, \mathrm{ZnO}, \mathrm{WO}_{3}$, $\mathrm{SnO}_{2}$, and so on. ${ }^{1-5]} \mathrm{TiO}_{2}$ has good optical and electronic properties, such as low cost, high catalytic performance, stable chemical properties and low toxicity. It has been widely studied and applied to the degradation of various pollutants. In order to improve the shortcomings, many scholars have made a modification of the titanium dioxide. First, the researchers mixed the $\mathrm{N}$ element and shortened the band-gap successfully. ${ }^{[6]}$ Others found that the doping of Fe ions can significantly decrease the recombination rate of the hole. ${ }^{[7]}$ Furthermore, someone added bentonite to $\mathrm{TiO}_{2}$, the result showed that the specific surface area, thermal stability, cation exchange property of $\mathrm{TiO}_{2}$ were significantly increased, and the catalytic efficiency was improved. ${ }^{[8]}$

In this study, we modify the titanium dioxide using other materials and the light utilization rate and photocatalytic activity of $\mathrm{TiO}_{2}$ is improved. We also apply it in the disposal of styrene in the river and achieved good results. In addition, the catalytic effect of various catalysts was compared, and the effect of various conditions on the reaction is researched.

\section{Experimental Procedure}

\section{Materials Preparation}

Tetrabutyl titanate $(24.0 \mathrm{ml})$ is doped into the mixture of $4.0 \mathrm{ml}$ glacial acetic acid and $90.0 \mathrm{ml}$ absolute ethyl alcohol. Keep agitate and get solution A. Then use 1.0ml hydrogen nitrate to mix up with $10.0 \mathrm{ml}$ deionized water and get solution B. Solution B is dropped into solution A slowly and keep agitate. Later, the transparent sol will form. Drop urea into the mixture $(\mathrm{Ti} / \mathrm{N}=2)$ slowly and keep agitate. Then use the same procedure with 2.1 to get products. The $\mathrm{N}-\mathrm{TiO}_{2}$ photo-catalyst was obtained. Use the same method to get $\mathrm{Fe}-\mathrm{TiO}_{2}$ photo-catalyst, Bentonite- $\mathrm{TiO}_{2}$ photo-catalyst using iron (III) nitrate $(\mathrm{Ti} / \mathrm{Fe}=5)$ and $20 \mathrm{ml}$ bentonite solution. All the samples are calcined at $600^{\circ} \mathrm{C}$. 


\section{Material Characterization}

\section{$X R D$}

The XRD results of $\mathrm{TiO}_{2}$ (calcined at $600^{\circ} \mathrm{C}$ ) and modified $\mathrm{TiO}_{2}$ including $\mathrm{Fe}-\mathrm{TiO}_{2}, \mathrm{~N}-\mathrm{TiO}_{2}$ and Bentonite- $\mathrm{TiO}_{2}$ are showed in Figure 1. The diffraction peak of $\mathrm{TiO}_{2}$ is relative to the standard X-ray of $\mathrm{TiO}_{2}$, and the crystal phase of the film is anatase. It has a high (101) orientation, which indicates that $\mathrm{TiO}_{2}$ has formed a certain ordered crystal structure. According to the X-ray line width method, the particle size of anatase $\mathrm{TiO}_{2}$ particles in the film was about $15 \mathrm{~nm}$. The crystal of 15 $\mathrm{nm}$ anatase phase has photo-catalytic activity, and it is also a prerequisite for obtaining smooth film.

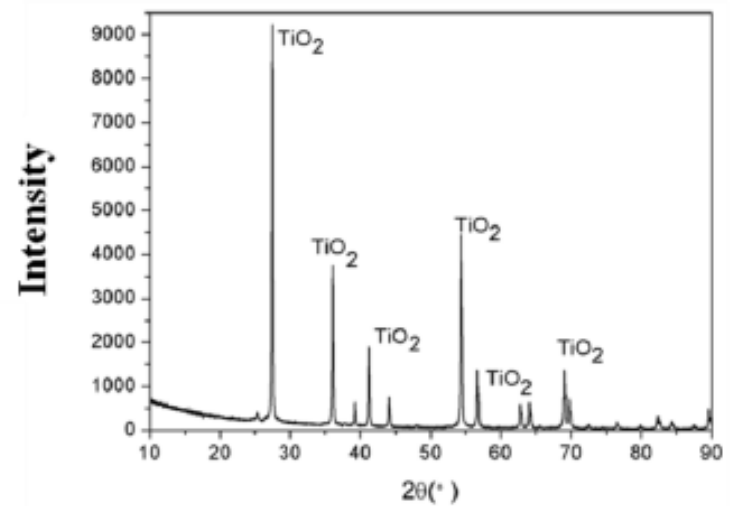

(a) $\mathrm{TiO}_{2}$

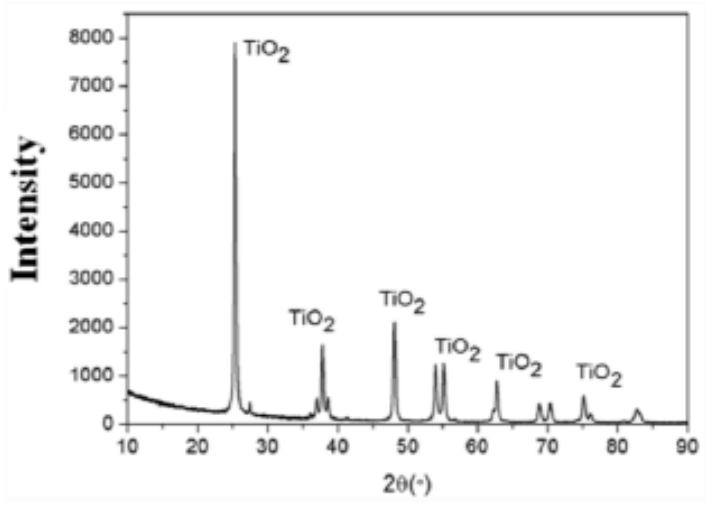

(c) $\mathrm{N}-\mathrm{TiO}_{2}$

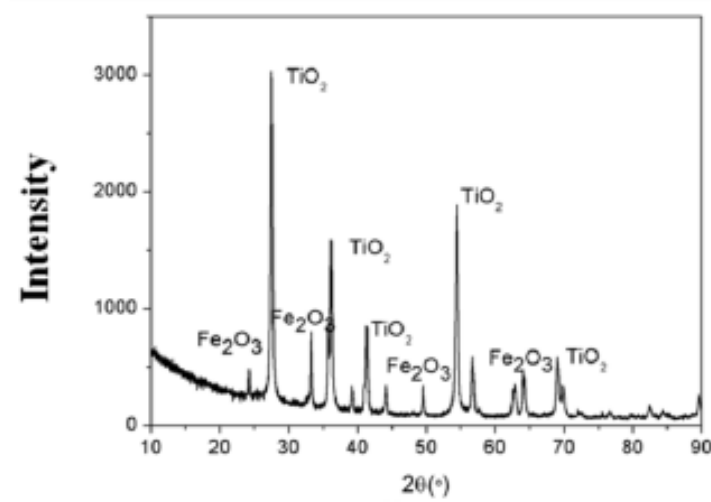

(b) $\mathrm{Fe}-\mathrm{TiO}_{2}$

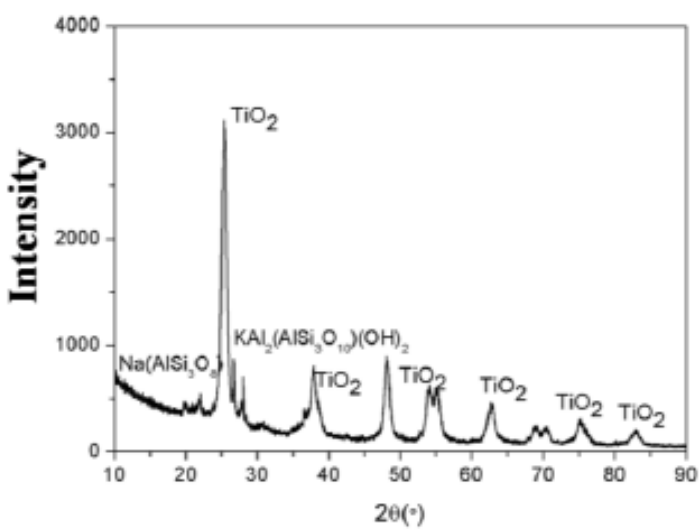

(d) Bentonite- $\mathrm{TiO}_{2}$

Figure1 The XRD results of different photocatalysts

\section{BET}

The results show that the specific surface area of $\mathrm{TiO}_{2}$ is gradually decreased with the increase of calcination temperature. But the surface area increased after other materials doped. In particular for bentonite- $\mathrm{TiO}_{2}$, the surface area almost reached the level of commercial titanium dioxide. So the specific surface area of doped photo-catalyst material is more significantly improved than single $\mathrm{TiO}_{2}$.

\section{SEM}

The experimental results show that the particles of care gradually increased as the calcination temperature increasing. The product of $\mathrm{TiO}_{2}$ is more uniform, but with the increase of calcination temperature, the particles of $\mathrm{TiO}_{2}$ are obviously uneven and the particles are obviously increased. And the particles of other doped materials which calcined at 600 degree are more uniform, smooth and smaller. It reveals that crystal structure of the doped photo-catalyst material are uniform than single $\mathrm{TiO}_{2}$, and it may improve the catalytic effect of photo-catalyst. Of course, the particle 
morphology of different doping samples is different.

\section{Photo-catalytic Degradation Reaction}

The styrene solutions of different concentration are prepared respectively. Then different kinds of catalysts are added into styrene solution. At the same time, keep agitate and open the UV lamp. Finally, the samples are centrifugal separated at different time. The level of styrene is measured by purge and trap GC-MS.

\section{Results and Discussion}

\section{Different Photocatalysts}

In this experiment, the water solution of the catalytic material is added to the pure styrene solution and keep agitate at room temperature. At the same time, the ultraviolet light is turned on. Get the reaction liquid at $0,5,10,20,40,60$ and 80 minutes, then centrifugal for 3 minutes and take the supernatant. The styrene concentration is determined by gas chromatography-mass spectrometry. The initial $\mathrm{pH}$ value of the solution is about 7.5, and the $\mathrm{pH}$ value of the solution is $6.5 \pm 0.3$ after the reaction.

The result shows out that styrene degradation rate increased as the reaction time increasing generally in Figure 2. And the degradation efficiency of doped $\mathrm{TiO}_{2}$ is better than $\mathrm{TiO}_{2}$ and the best is bentonite- $\mathrm{TiO}_{2}$. As the results of previous studies, nitrogen doping can increase the absorption of visible light in our research. Nitrogen entering into the titanium dioxide lattice can move its absorption edge to the visible range. This is because the Nitrogen doping could produce an intermediate band gap to shorten the gap in the valence band top. For $\mathrm{Fe}^{3+}$ doped $\mathrm{TiO}_{2}$, it shows a strong visible light absorption. This is because $\mathrm{Fe}^{3+}$ doping can form an impurity level in the band gap of $\mathrm{TiO}_{2}$. The bentonite can absorb a part of the visible light and its big specific surface area is also benefit to the reaction. The result also confirms that the effect of modified photo-catalyst is better than pure $\mathrm{TiO}_{2}$.

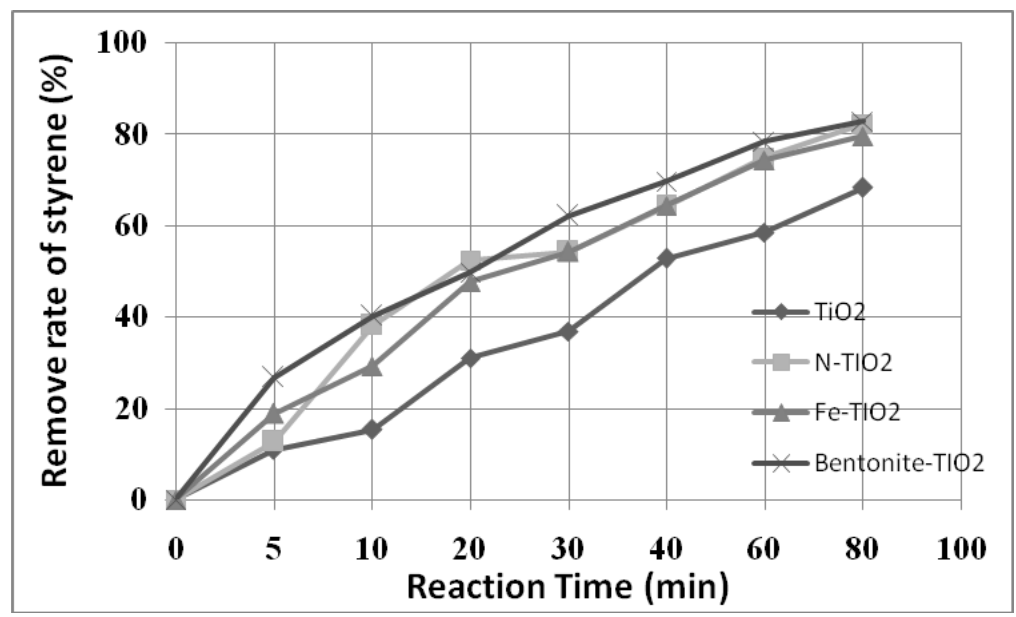

Figure 2 The results of using different photo-catalysts

\section{The Influence of Lighting Time}

In this part, we use the best materials of Bentonite- $\mathrm{TiO}_{2}$ which selected from the above experiment. The experiment procedure is basically the same as 3.1, but the UV irradiation time is $0,20,40$, $60,80 \mathrm{~min}$. The result reveals that styrene degradation rate increased as the reaction time and also lighting time increasing in Figure 3. Generally, the degradation rate increased significantly at the beginning. After the illumination time is up to 60 minutes, although the photolysis rate is still increasing, the trend is tends to be gentle. Researcher suggests that the wettability can be changed 
under UV irradiation no matter polycrystalline or single crystal surfaces of anatase and rutile. ${ }^{\text {[9] }}$ With the increase of illumination time, the contact angle of the membrane and water gradually becomes smaller, and the catalytic activity increases gradually. When it up at a certain time, the contact angle will not decrease as the light time increasing, thus the catalytic activity of the membrane becomes stable. As the lighting time increasing, the catalytic activity increases gradually. After a certain time, the catalytic activity will be stable.

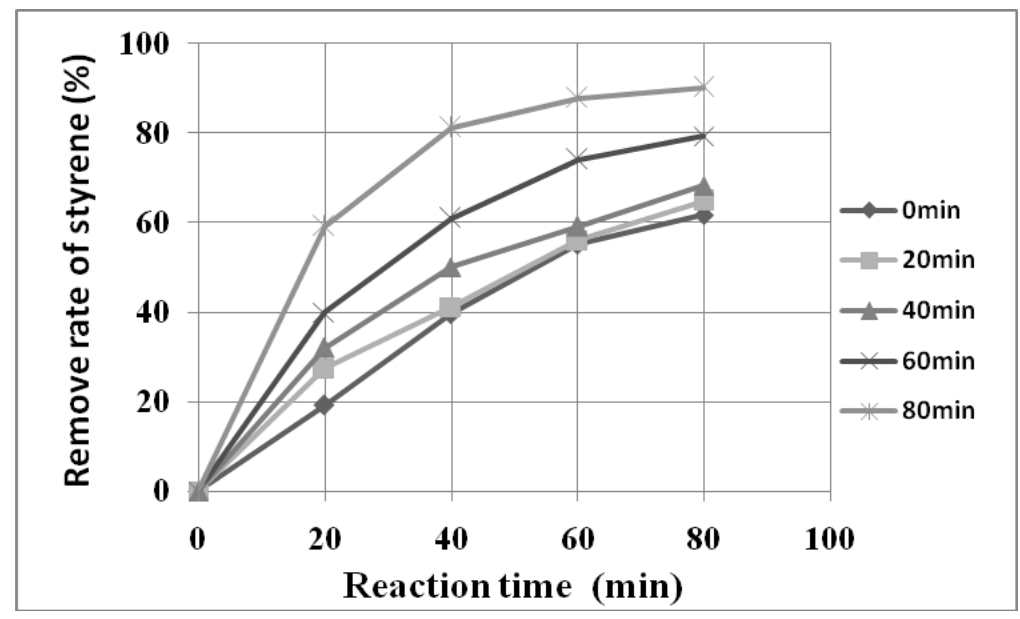

Figure 3 The influence of lighting time

The ultraviolet light emitted by the high pressure mercury lamp is stronger than natural light, so the photon energy is greater than or equal to the band gap energy of $\mathrm{TiO}_{2}$. When the light radiate to the $\mathrm{TiO}_{2}$, the electronics in the valence band can be excited into the conduction band to generate electron-hole pairs, and a series of reaction will take place on the surface. Finally, the free radicals will be generated, so that the organic compound will be degraded to $\mathrm{CO}_{2}$ and $\mathrm{H}_{2} \mathrm{O}$. ${ }^{[10-13]}$ when the photon energy is stronger, the degradation effect is more obvious. So the photo-degradation rate of chemicals is higher under mercury lamp than sunlight. But in the practical application, it is feasible to use solar energy and it is also to the economic point.

\section{The Influence of Reaction Temperature}

In this part, experiments of different reaction temperatures were carried out using the best materials selected in the former experiment. The method is the same as above, but the reaction temperature is 25 degrees and 35 degrees. The removal effect of photocatalyst in different reaction temperatures is shown in Figure 4. The result shows that the chemical removal effect is better under reaction temperature of 35 degrees than 25 degrees. As the degradation reaction is endothermic reaction, the temperature rise will inevitably accelerate the reaction. It can be concluded that when the chemical leakage occurs in the condition of high temperature, the effect of photocatalytic degradation of chemicals will be more obvious. 


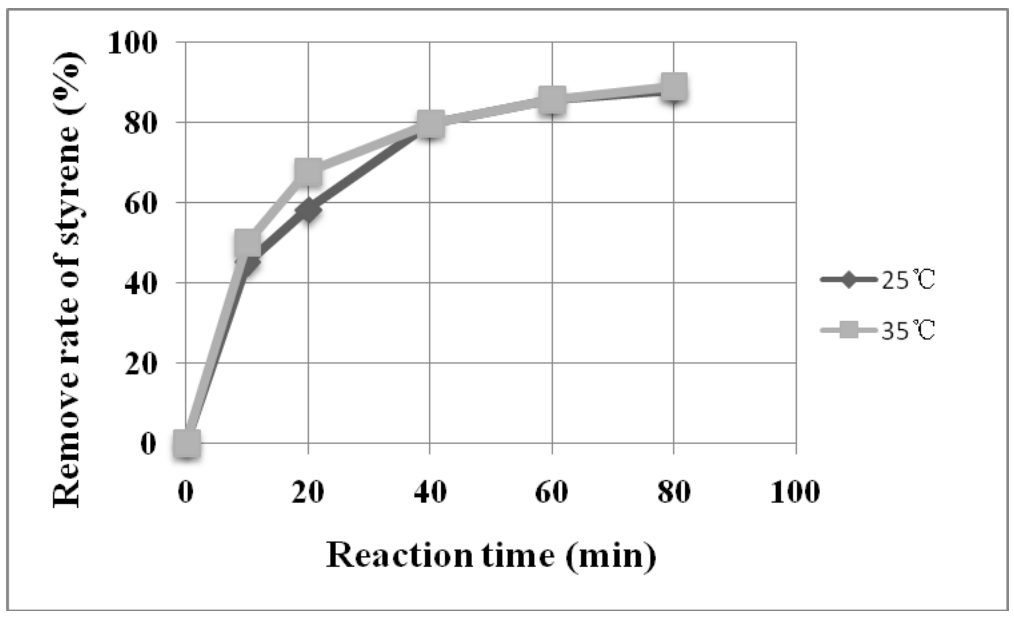

Figure 4 The influence of reaction temperature

\section{The Influence of Concentration and Reaction Type}

Researchers suggest that the degradation rate was related to the initial concentration of reactants. The initial concentration of styrene in solution is inversely proportional to the rate of photolysis. It reveals that the photocatalytic technology is an effective method to deal with the low content of organic pollutants. The effect of the concentration of reactant on the degradation rate is similar to the effect of the intensity of the light intensity. The rate equation of heterogeneous photocatalytic degradation can be expressed as the Langmuir-Hinshelwood kinetic equation. The kinetic equation of the photocatalytic reaction shows that when the reaction concentration is low, the reaction is in accordance with the law of first order kinetics, that is, the degradation rate is proportional to the concentration. When the reactant concentration increased to a certain degree, the reaction rate increased with the increase of the concentration of the reactants. But this increase is not proportional to the concentration of reactants. Then, as the increase of concentration, the reaction rate will not change with the concentration.

\section{Conclusions}

In this work, the doped $\mathrm{TiO}_{2}$ is applied in styrene photo-catalytic remediation. Different kinds of catalysts were used, and the degradation rate of styrene was researched under different conditions. The results revealed that the styrene removal percentage increased as the reaction time increasing generally. For the four types of catalysts, the efficiency of bentonite- $\mathrm{TiO}_{2}$ is the best. Furthermore, the styrene degradation rate increased as the lighting time increasing. Finally, the styrene degradation rate was accelerated as the reaction temperature increasing.

When some organic material has a strong UV absorption, high concentration of organic matter will shield the ultraviolet radiation of the catalyst, which can affect the photocatalytic efficiency. Therefore, the photocatalytic method is suitable for the treatment of slightly polluted water. In inland chemicals leakage accident treatment, other methods should be used first to reduce pollutant content and then use photocatalyst method to treat rest of pollutants for the economic point. Thus, the effect of water treatment and the economic benefit will be improved obviously.

\section{Acknowledgements}

This study was supported by the Prospective Basic Research Project of China Waterborne Transport Research Institute (WTI61421, WTI61607) and the Youth Fund Project of China Waterborne Transport Research Institute (WTI61426). 


\section{References}

[1] T. Leshuk, R. Parviz, P. Everett, et al. Photocatalytic Activity of Hydrogenated $\mathrm{TiO}_{2}$. ACS Appl. Mater. Interfaces, 2013, 5 (6), 892-1895.

[2] Z. R. Tang, X. Yin, Y. H. Zhang, et al. Synthesis of Titanate Nanotube-CdS Nanocomposites with Enhanced Visible Light Photocatalytic Activity. Inorg. Chem., 2013, 52 (20), 11758-11766.

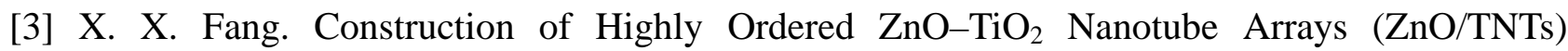
Heterostructure for Photocatalytic Application. ACS Appl. Mater. Interfaces, 2012, 4 (12), 7055-7063.

[4] H. Tada, A. Kokubu, M. Iwasaki, et al. Deactivation of the $\mathrm{TiO}_{2}$ Photocatalyst by Coupling with $\mathrm{WO}_{3}$ and the Electrochemically Assisted High Photocatalytic Activity of $\mathrm{WO}_{3}$. Langmuir, 2004, 20 (11), 4665-4670.

[5] S. Wu, H. Q. Cao, S. Yin, et al. Amino Acid-Assisted Hydrothermal Synthesis and Photocatalysis of $\mathrm{SnO}_{2}$ Nanocrystals. J. Phys. Chem. C, 2009, 113 (41), 17893-17898.

[6] J. Fang, F. Wang, K. Qian, et al. Bifunctional N-Doped Mesoporous $\mathrm{TiO}_{2}$ Photocatalysts. J. Phys. Chem. C, 2008, 112 (46), 18150-18156.

[7] D. K. Wang, M. T. Wang, and Z. H. Li. Fe-Based Metal-Organic Frameworks for Highly Selective Photocatalytic Benzene Hydroxylation to Phenol. ACS Catal., 2015, 5, 6852-6857.

[8] J. Y. Feng, X. J. Hu, and P. L. Yue. Novel Bentonite Clay-Based Fe-Nanocomposite as a Heterogeneous Catalyst for Photo-Fenton Discoloration and Mineralization of Orange II. Environ. Sci. Technol., 2004, 38 (1), 269-275.

[9] Fujishima, T. Inoue, K. Honda. Competitive photoelectrochemical oxidation of reducing agents at the titanium dioxide photoanode. J. Am. Chem. Soc., 1979, 101 (19), 5582-5588

[10] H. Jiang, L. Gao. Enhancing the UV Inducing Hydrophilicity of $\mathrm{TiO}_{2}$ Thin Film by Doping Fe Ions. Materials Chemistry and Physics, 2002, 77, 878-881.

[11] T. Ohno, F. Tanigawa, K. Fujinara, et al. Photocatalytic oxidation of Water by Visible Light Using Ruthenium-Doped Titanium Dioxide Powder. Journal of Photochemistry and Photobiology A: Chemistry, 1999, 127, 107-110.

[12] S. J. Kim, H. G. Lee, J. K. Lee, et al. Photoredox Properties of Ultrafine Rutile $\mathrm{TiO}_{2}$ Acicular Powder in Aqueous 4-ChloroPhenol, Cu-E DTA and Pb-EDTA Solutions. Applied Catalysis A: General, 2003, 242, 89-99.

[13] V. M. Cristante, A. B. Araujo, S. M. A. Jorge, et al. Enhanced Photocatalytic Reduction of Hg (II) in Aqueous Medium by 2-Aminothiazole-Modified $\mathrm{TiO}_{2}$ Particles. Journal of the Brazilian Chemical Society, 2006, 17, 453-457. 